\title{
Effectiveness of Prenatal Ultrasound Examination at Second Trimester in Detecting Fetal Congenital Abnormalities-A Preliminary Study
}

\author{
Madan Thapa $^{1}$, Shyam Sundar Parajuly ${ }^{\# 1}$, Rajesh Adhikari ${ }^{2}$, Manish Kiran Shrestha ${ }^{3}$ \\ ${ }^{1}$ Department of Radiology, Western Regional Hospital, Pokhara Academy of Health Sciences \\ ${ }^{2}$ Department of Obstetrics and Gynecology, Western Regional Hospital, Pokhara Academy of Health Sciences \\ ${ }^{3}$ Department of Radiology, Gandaki Medical College, Pokhara, Nepal
}

\section{${ }^{\# 1}$ Correspondence:}

Dr. Shyam Sundar Parajuly, PhD

Department of Radiology, Ultrasound Unit

Western Regional Hospital,

Pokhara Academy of Health Sciences

Email: shyampkr@gmail.com

Article received : $15^{\text {th }}$ July, 2019

Article accepted : $25^{\text {th }}$ September, 2019

\section{ABSTRACT}

Objective: Anomaly scan at the second trimester provides the detail anatomical study of fetus. Any structural or morphological defects detected prenatally guides the parents and doctors for further counseling. The main objective of this study was to detect the fetal congenital anomalies in high risk patients at 18-22 weeks and highlight the effectiveness of prenatal ultrasound examination. Materials and Methods: This was a hospital based descriptive study done during the period of 2017 January to 2019 January in the department of Radiology, ultrasound unit, Pokhara Academy of Health Sciences, Nepal. Pregnant women who were first examined and evaluated by the Obstetrician during the ante-natal check up (ANC) either by asking the patient history or by mother age or any symptoms or chance of being abnormalities or high risk patients during that time frame (18-22 weeks) were enrolled for the study. Maternal age, parity, any history of previous anomalies, previous history of miscarriage/Intra Uterine Fetal Demise ( IUFD) or any exposure to radiation or drugs, history of other disease were recorded during the filling of consent form. High risk patients were identified by the Obstetrician and anomaly examination was prescribed at second trimester (18-20 weeks). Descriptive analysis was done using SPSS 20. Results:There were two hundred and twenty four patients who had undergone anomaly (targeted fetal anatomy) examination which were referred for "anomaly scan" from the gynecology and obstetrics department during that period. Among all the cases, fourteen cases had anomalies detected during the scan (18-22 weeks). Out of which seven cases had central nervous system related anomalies, two cases had skeletal deformities (dysplasia), two case had urinary tract related anomalies, one had lungs related anomalies and one had single umbilical artery with polyhydroamnios associated with duodenal atresia and one case had malformation of lymphatic system ( cystic hygroma) Conclusion:Our study revealed that ultrasound scan performed between 18-22 weeks of pregnancy is effective in diagnosing major fetal abnormalities in the high-risk patients.

Keywords: Anomaly scan, Congenital anomalies, Second trimester, Prenatal, Ultrasound. 
Original Article

\section{INTRODUCTION}

Second trimester scan is done between 18 and 22 weeks for detailed anatomical evaluation. ${ }^{1}$ Among the indicated ultrasound examination in pregnancy, the second trimester targeted anomaly scan can be considered the most important, and a thorough anatomical examination of the fetus is warranted. ${ }^{1,2}$ It is well known that congenital anomalies are among the leading causes of perinatal and infant morbidity and mortality. They are present in 14 percentage of newborns with major anomalies in $2-5 \%$ accounting for $20-30 \%$ of perinatal deaths. ${ }^{3-5}$ Fetal anomalies that are undetectable in first trimester are detectable in second trimester. Second trimester ultrasound examination between eighteen and twenty two weeks is the standard for fetal anatomical evaluation. ${ }^{6,7}$ Abnormal neonates causes psychological trauma to the parents and cost for care is greater because of attendant morbidities of late diagnosis. Prenatal ultrasound (US) screening can detect several anomalies, about $90 \%$ of which occurs in fetuses born to parents with unrecognizable risk factors. ${ }^{4}$ Detection of structural fetal anomalies is dependent on numerous factors, such as maternal and gestational ages, skill and experience of sonographers/radiologists as well as the quality and resolution of the equipment used. ${ }^{8,9}$

The International Society of Ultrasound in Obstetrics and Gynecology (ISUOG) has developed a practice guidelines and consensus statements as educational recommendations. According to them every pregnant women should be offered an US scan to screen for fetal systemic anomalies between the 18-20 weeks of gestation by a well trained sonographer/radiologist with the appropriate equipment settings. ${ }^{10}$ The overall aim of fetal anomaly screening is to identify potential problems so that parents can make informed choices as well as to improve the safety of birth. Many of the scientific paper published revealed that second trimester US shows high specificity for identifying fetal anomalies. ${ }^{11,12}$

Fetal anomaly scan/screening has been regularly well established and practiced in developed countries;
Effectiveness of Prenatal Ultrasound Examination at Second Trimester. Thapa M et al. however such practice in developing countries like Nepal is just emerging. To the best of our knowledge, there are no standardized national guidelines for the US screening of pregnant women with high risk pregnancies for congenital anomalies in Nepal.

In resource-poor countries where expensive invasive procedures such as amniocentesis may not be costeffective or readily available, ultrasound imaging may be the only affordable modality for determining a woman's risk of delivering a chromosomally abnormal baby who is more likely to die in the perinatal period than a chromosomally normal baby, so that they might consider termination. Any pregnant women with having high risk of getting fetal abnormalities should consult the Obstetrician and go for anomaly scan. The aim of the study was to measure the effectiveness of anomaly scan at 18-22 weeks in detecting congenital abnormalities in high risk patients.

\section{MATERIALS AND METHODS}

This was a hospital based descriptive study done during the period of 2017 January to 2019 January in the Department of Radiology, Ultrasound Unit, Pokhara Academy of Health Sciences (PoAHS), Nepal. PoAHS was former called as Western Regional Hospital, which is also a tertiary hospital covering a population of about 2.4 million of Gandaki Province. Patient who were first examined and evaluated by the Obstetricians and among them who were at high risks were only advised to go for "anomaly scan" and those who had given written informed consent were enrolled in this study. Two experts with more than four years of experiences in fetal scan performed all the scans. The mean time of 30 minutes was allocated for each scan. All the patients undergoing the procedure were appointed to come early morning in the first hour of outpatient department of Radiology. No any preparations were required. They were asked to bring a visitor/ relative for possible counseling and pitfalls. Transabdominal ultrasound examination using the obstetrics setting in Mindray DC93 (machine) was performed. Each image was saved in 
the machine. Anatomical systemic evaluation of fetus were done using the ISUOG guidelines for performing the mid-trimester US scans. ${ }^{10}$ After discussion with the obstetricians about the detected anomalies, parents/close relatives were counseled. Mothers were counseled for termination of pregnancy in case of major abnormalities. In case of minor abnormalities follow up US scan was advised. Maternal age, parity, any history of previous anomalies detected, exposure to any radiation or drugs, history of other disease were recorded during the filling of consent form. Ethical clearance was obtained from the hospital ethical committee.administration. Descriptive analysis was done by using SPSS version 20 .

\section{RESULTS}

According to the hospital registry there were approximately 10,000 women who came for antenatal checkup irrespective of the trimester during the period in our centre. Patient who underwent anomaly scan with appointment and registered with their information in the Department of Radiology, were two hundred and twenty four. Age group distribution of mothers who went anomaly scan can be seen in figure 1 .

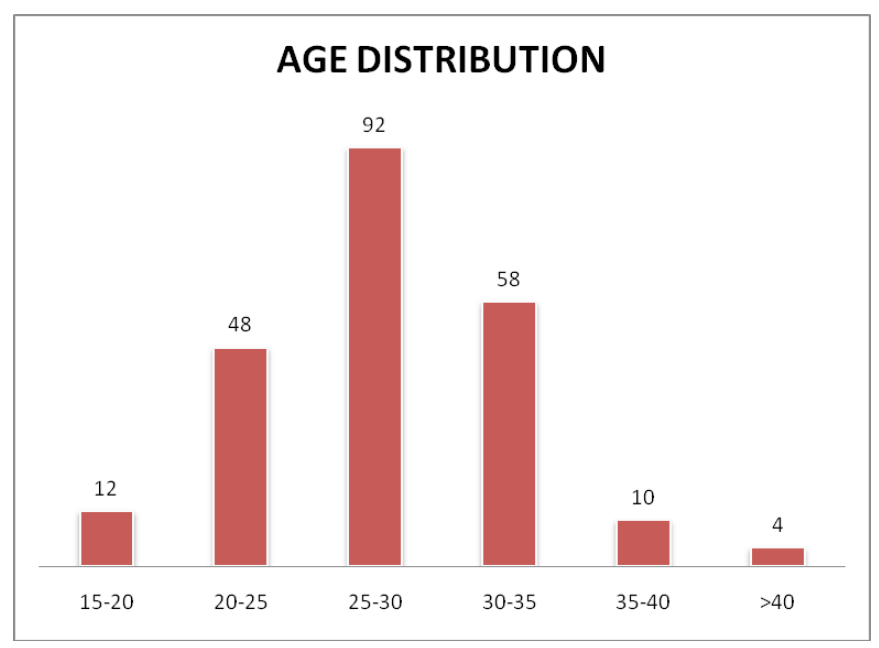

Figure 1. Age distribution among the different groups pattern

Among 224 cases, 110 women (49.11\%) were primi and $114(50.89 \%)$ were multiparous. Out of them, 152 (67.85\%) mothers were below 30 years of age and 72
$(32.14 \%)$ were more than 30 . Two cases had anomalies detected in younger age group ( less than 30 years ) and 12 cases had anomalies in age group more than 30 years of age, Of the 114 multiparous, five cases had previous history of pregnancy with IUFD (Intra uterine fetal Demise). The causes of IUFD were severe oligohydroamnios (three) cases), anencephaly( one case) and gross hydrocephalus(one case).

$14(6.25 \%)$ anomalies were detected in this study. Among the detected anomalies, there were seven (50\%) cases involving central nervous system (CNS) that includes (one anencephaly; three meningomyelocele and three severe ventriculomegaly ) two cases had skeletal deformities (dysplasia), two case had urinary tract related anomalies, one had lungs related anomalies and one had single umbilical artery with polyhydroamnios associated with duodenal atresia, one case had malformation of lymphatic system ( cystic hygroma) as shown in figure 2. Among the cases involving central nervous system, one case had severe neural tube defect (anencephaly) associated with abdominal wall defect with protrusion of intestinal loops into the amniotic fluid (Gastrochisis). The same case also presented skeletal deformity ( left leg club foot) as presented in figure 3 .

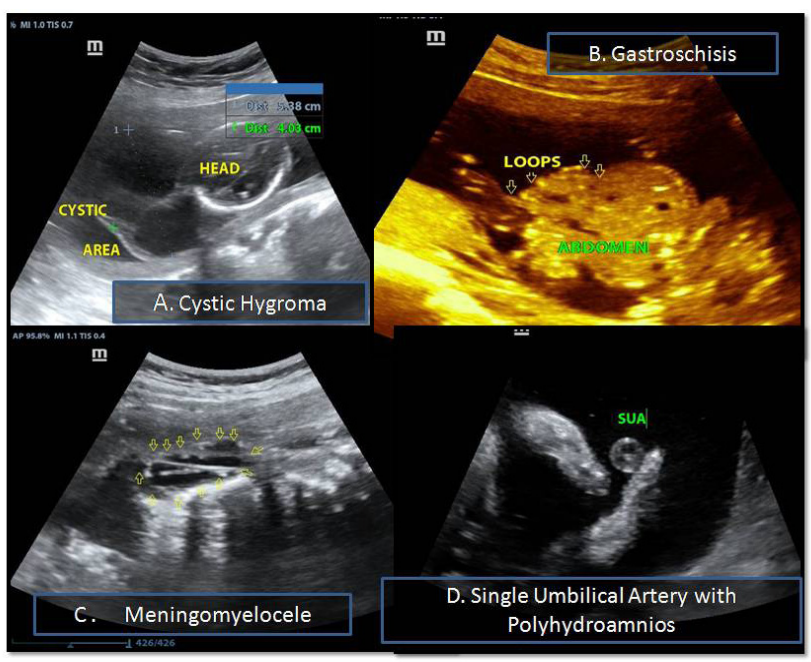

Figure 2. Illustarted images demonstrated other major malformations Cystic Hygroma(A), Gastroschisis ( B) and Meningomyelocele (C) in different patients during the 18-22 scan respectively. 


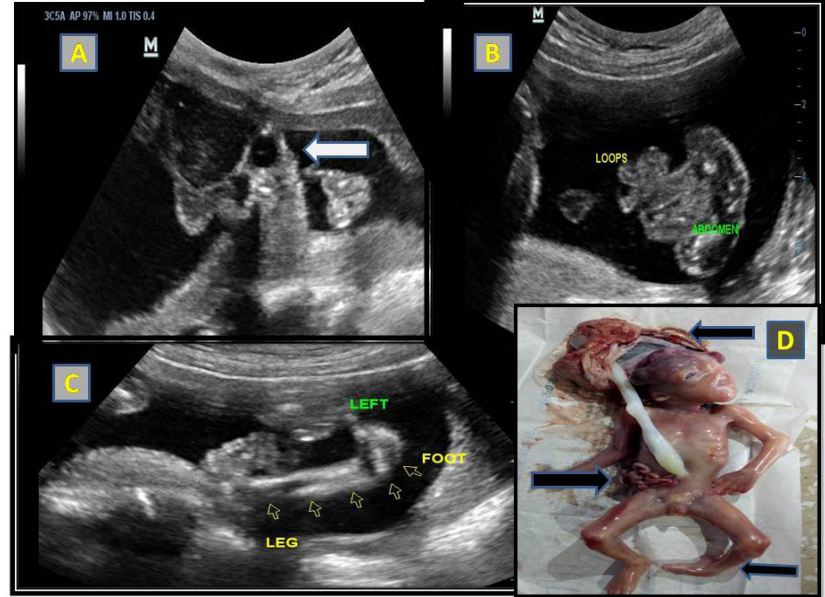

Figure 3. Illustrated figure showed absent of bony calvaria with "frogs eye" sign (Arrow head) A in 19 weeks of pregnant women; Protrusion of bowel loops from the defect of abdominal wall $B$; left leg with club foot showing inward rotated foot (C); Pictograph showing exposed brain tissue, exposed intestinal loops and left leg with club foot (Arrowheads).

\section{DISCUSSION}

Two hundred and twenty four cases were precisely asked for fetal anomaly scan by the obstetricians over a 2 -year period, and were evaluated. The incidence of congenital anomalies detected prenatally by US at $18-22$ weeks is $0.14 \%$ which is lower than the study conducted by Bricker et al (1.16\%). ${ }^{13}$ Globally, Congenital malformations affect approximately $2-3 \%$ of all live births every year. ${ }^{14}$ Study conducted in maternity hospital of Kathmandu revealed the incidence rate of congenital malformations is $0.36 \%$ of them $40 \%$ cases had CNS malformations 15 which is quite similar to our study, 50\%. Cardiac anomalies were not detected in our study, it may be due to lack of expertise in fetal echocardiography and lack of high definition US machine available in our hospital. Besides most of the government hospitals in rural areas of Nepal do not practice anomaly scan in their facilities. The reason may be antenatal caregivers are unaware of this service and don't routinely request for prenatal anomaly screening outside the fetal well being scans, the same scenario can be seen even in tertiary hospitals. ${ }^{16}$ Few research showed that preconceptional supplement of folic acid reduces the neural tube defect and other severe anomalies. ${ }^{17}$ This kind of clinical practice is not seen in our population. However, the country has made significant progress in preventing congenital anomalies through programs such as folic acid supplementation during early pregnancy (first trimester).

The commonest malformations in the country are cleft lip and palate, neural tube defects and congenital heart diseases. ${ }^{18}$ The South-East Asia Regional Neonatal Perinatal database (SEAR NPD/WHO Report, 20072008) reported that congenital malformations accounted for $11.1 \%$ of primary causes of neonatal death. ${ }^{5}$ Besides incidence of congenital anomalies in our study group was more in older maternal age group ( $>30$ years) 12 cases $(85.7 \%)$ which is quite similar to other study indicating that increasing with the maternal ages increases the risk for congenital malformations. ${ }^{19,20}$

\section{CONCLUSION}

Our findings suggest that anomaly scan at 18-22 weeks for high risk patients are highly effective in detecting fetal malformations.

\section{LIMITATIONS}

As this was the preliminary study done in our institute which may not reflect the results representing the entire nation. Only high risk patients which were precisely advised for anomaly scan by the Obstetricians were enrolled for the study which might have created biasness between the population which are not at risk. Similarly we did not evaluate the intraobserver and interobserver variabilty. More, risk factors like consanguinity of marriage were not recorded. Chromosomal anomalies could not be included due to lack of resources available.

\section{RECOMMENDATION}

If possible every woman (low risk/high risk) in this period of gestation (18-22 weeks) should go detailed fetal anatomical examination. An early detection anomaly offers parents the option of an earlier termination of pregnancy after counseling with the obstetricians. More, prenatal US scan at 11-14 weeks helps in early detection of anomalies like anencephaly. Government and local health policy makers should take the responsibility for providing the awareness to its people and service providers should upgrade the services and if possible the service should be provided in rural belt of the country. Government service providers have to make policy regarding the fetal anomaly scan and standard guidelines have to be followed for its long-term implementation. 
1. Suresh S, Suresh I. The second trimester obstetric scan $(7+3=10)$ : A rational approach (including the "rule of three"). J Fetal Med 2014; 1:59-73.

2. Premnath KPB, Joy B, Toms A, Sleeba T. Image Acquisition Adequacy for Second Trimester Targeted Fetal Scans - A Clinical Audit. Int J Sci Stud 2017; 5(3):57-60.

3. Dastgiri S, Stone DH, Le-Ha C, Gilmour WH. Prevalence and secular trend of congenital anomalies in Glasgow, UK. Arch Dis Child. 2002; 86:257-63

4. Akinmoladun JA, Ogbole GI, Lawal TA, Adesina OA. Routine prenatal ultrasound anomaly screening program in a Nigerian university hospital: Redefining obstetrics practice in a developing African country. Niger Med J. 2015;56(4):263-67.

5. WHO South East Asia Regional Neonatal-Perinatal Database Report 2007-2008. Available from http:// ghdx.healthdata.org/record/who-south-east-asiaregional-neonatal-perinatal-database-report-2007-2008. Accessed July 72019.

6. Syngelaki A, Chelemen T, Dagklis T, Allan L, Nicolaides KH. Challenges in the diagnosis of fetal nonchromosomal abnormalities at 11- 13 weeks. Prenatal Diagnosis. 2011;31(1):90- 102.

7. Srisupundit K, TheeraTongsong T, Sirichotiyakul S, Chanprapaph P. Fetal structural anomaly screening at 11-14 weeks of gestation at Maharaj Nakorn Chiang Mai Hospital. J Med Assoc Thai. 2006; 89(5):588-93.

8. Ewigman BG, Crane JP, Frigoletto FD, LeFevre ML, Bain RP, McNellis D. Effect of prenatal ultrasound screening on perinatal outcome. RADIUS Study Group. N Engl J Med. 1993;329:821-27.

9. Grandjean H, Larroque D, Levi S. The performance of routine ultrasonographic screening of pregnancies in the Eurofetus Study. Am J Obstet Gynecol. 1999;181(2):446-54. 10.Salomon LJ, Alfirevic Z, Berghella V, Bilardo C, Hernandez-Andrade E, Johnsen SL, et al. Practice guidelines for performance of the routine midtrimester fetal ultrasound scan. Ultrasound Obstet Gynecol. 2011;37(1):116-26.
11. Nakling J, Backe B. Routine ultrasound screening and detection of congenital anomalies outside a university setting. Acta Obstet Gynecol Scand. 2005; 84(11):1042-48.

12. Nikkilä A, Rydhstroem H, Källén B, Jörgensen C. Ultrasound screening for fetal anomalies in southern Sweden: A population-based study. Acta Obstet Gynecol Scand. 2006; 85(6):688-93.

13.Shrestha S, Dwa Y, Jaiswal P \& Parmar B. Congenital anomalies in antenatal ultrasound scan at a tertiary care teaching hospital. Journal of Patan Academy of Health Sciences. 2018; 5(1):26-30

14.Kashyap N, Pradhan M, Singh N, Yadav S. Early Detection of Fetal Malformation, a Long Distance Yet to Cover! Present Status and Potential of First Trimester Ultrasonography in Detection of Fetal Congenital Malformation in a Developing Country: Experience at a Tertiary Care Centre in India. J Pregnancy. 2015; 2015:623059

15. Malla BK One year review study of congenital anatomical malformation at birth in Maternity Hospital (Prasutigriha), Thapathali, Kathmandu. Kathmandu Univ Med J. 2007; 5(4):557-60.

16. Akinmoladun JA, Ogbole GI, Lawal TA, Adesina OA. Routine prenatal ultrasound anomaly screening program in a Nigerian university hospital: Redefining obstetrics practice in a developing African country. Niger Med J. 2015; 56(4):263-67.

17. De-Regil LM, Peña-Rosas JP, Fernández-Gaxiola AC, Rayco-Solon P.Effects and safety of periconceptional oral folate supplementation for preventing birth defects. Cochrane Database of Systematic Reviews 2015, 12: CD007950.

18. Birth Defects In South-east Asia A Public Health Challenge Situation Analysis WHO 2013;2(1):75

19.Sugunbai NS, Mary M, Shymalan K, Nair PM. An etiological study of congenital malformations in newborns. Indian Pediatr. 1982; 19:1003-09.

20. Mohanty C, Mishra OP, Das BK, Bhatia BD, Singh G. Congenital malformations in newborns: A study of 10,874 consecutive births. J Anat Soc India. 1989;38:101-11. 